\title{
Intrathecal catheter for severe low back pain during deep brain stimulation placement: illustrative case
}

\author{
Bryan Kunkler, MD, ${ }^{1}$ Alan Tung, MD, FRCPC, ${ }^{1}$ Parag G. Patil, MD, PhD, ${ }^{2}$ Srinivas Chiravuri, MD, ${ }^{1}$ and Vijay Tarnal, MD, $\mathrm{FRCA}^{1}$ \\ Departments of ${ }^{1}$ Anesthesiology and ${ }^{2}$ Neurological Surgery, University of Michigan Medical School, Ann Arbor, Michigan
}

BACKGROUND Deep brain stimulation (DBS) is a U.S. Food and Drug Administration-approved therapy for medically refractory Parkinson's disease, essential tremor, and other neurological conditions. The procedure requires prolonged immobility and can result in significant patient discomfort, potentially limiting patient selection. In addition, surgical requirements necessitate avoidance of medications that may alter or suppress the patient's arousal or baseline tremor during macrostimulation testing.

OBSERVATIONS In this study, the authors describe the use of continuous spinal anesthesia with local anesthetic to manage a patient with severe back pain who was intolerant of semisupine position during stereotactic computed tomography and stage 1 of DBS placement.

LESSONS Continuous spinal anesthesia is an effective strategy to manage patients with severe back pain undergoing DBS surgery for upper extremity motor symptoms.

https://thejns.org/doi/abs/10.3171/CASE21285

KEYWORDS intrathecal catheter; deep brain stimulator; back pain

Deep brain stimulation (DBS) is a U.S. Food and Drug Administration (FDA)-approved therapy for medically refractory Parkinson's disease, essential tremor, and several other neurological conditions. In DBS surgery, an electrode is precisely placed into a targeted structure, and stimulation is applied to relieve patient symptoms. Contraindications to DBS surgery include conditions that increase the risk of operative complications or device malfunction (i.e., coagulopathy) and those that limit the expected benefits of DBS (i.e., dementia or unable to use the neurostimulator). ${ }^{1}$ For surgical positioning, patients are placed in a stereotactic headframe and positioned semisupine for the hours-long procedure. ${ }^{1}$ With up to $60 \%$ prevalence of back pain, ${ }^{2}$ patients with Parkinson's disease may not tolerate the discomfort of the long duration of immobility. ${ }^{1}$

In this study, we describe the use of continuous spinal anesthesia (CSA) with a local anesthetic to manage a patient with Parkinson's disease who had severe back pain and neurogenic claudication and was unable to tolerate DBS placement in a semisupine position.

\section{Illustrative Case}

A 77-year-old right-handed man with American Society of Anesthesiologists (ASA) physical status classification 3 and tremor-predominant Parkinson's disease was scheduled to undergo a left ventral intermediate nucleus DBS implant to manage his right-hand tremor. The patient had a history of severe axial lumbar back pain due to lumbar spinal stenosis, as well as a previous multiple L3-5 lumbar laminectomy and fusion. Because of his debilitating severe back and lower extremity neurogenic claudication pain, the patient was using a wheelchair and unable to be supine even for a short period of time. His medical history was also significant for atrial fibrillation treated with apixaban, congestive heart failure, coronary artery disease, mild aortic and mitral regurgitation, type 2 diabetes mellitus, and hypertension.

During the patient's first scheduled DBS procedure, severe back pain prevented him from remaining supine for stereotactic computed tomography $(\mathrm{CT})$, leading to the cancellation of the procedure. A

ABBREVIATIONS ASA = American Society of Anesthesiologists; CSA = continuous spinal anesthesia; CSF = cerebrospinal fluid; CT = computed tomography; $\mathrm{DBS}=$ deep brain stimulation; FDA = U.S. Food and Drug Administration; NRS = numerical rating scale. INCLUDE WHEN CITING Published July 19, 2021; DOI: 10.3171/CASE21285.

SUBMITTED May 7, 2021. ACCEPTED June 10, 2021

(C) 2021 The authors, CC BY-NC-ND 4.0 (http://creativecommons.org/licenses/by-nc-nd/4.0/). 


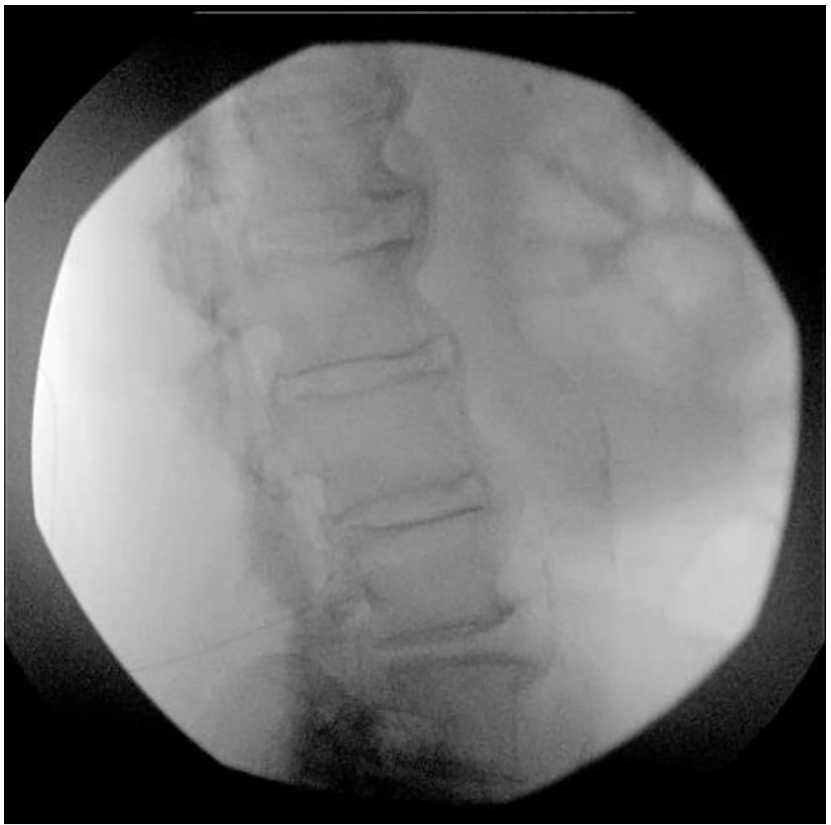

FIG. 1. Lumbar radiograph showing the tip of the intrathecal catheter at the level of $L 2$.

decision was made to use a CSA technique for the subsequently rescheduled stereotactic CT scans and stage 1 DBS (lead placement) and general anesthesia for stage 2 DBS (placement of the pulse generator). Both the stage 1 and stage 2 DBS procedures were scheduled for the same day. This titratable approach would help manage the patient's lower back and leg pain while allowing the patient to be fully alert and cooperative with the neurosurgical team during the stage 1 portion of the DBS procedure.

Preoperatively, apixaban was withheld per institutional guidelines. On the day of surgery, the patient was brought to the operating room for the placement of an intrathecal catheter and a Leksell stereotactic headframe (Elekta). Standard ASA monitors were placed on the patient. Using an aseptic technique, an intrathecal catheter was successfully placed under fluoroscopic guidance at the lumbar interspace level of $L 4-5$. There were no immediate complications after the catheter placement. The tip of the catheter was positioned at the level of $\mathrm{L} 2$ and confirmed using fluoroscopy (Fig. 1). After positive aspiration of cerebrospinal fluid (CSF), an initial dose of $0.5 \mathrm{~mL}$ of $0.5 \%$ isobaric bupivacaine was injected via an intrathecal catheter, following which no significant hemodynamic (heart rate and blood pressure) changes were noted. An additional $1 \mathrm{~mL}$ of $0.5 \%$ isobaric bupivacaine was injected without difficulty. A sensory band was identified by pinprick sensation (toothpick) and by response to cold (ice) corresponding to the eighth thoracic segment (T8). A Leksell stereotactic headframe was applied, and CT scan was performed. The anesthesia team was physically present for the duration of imaging.

Before the placement of the intrathecal catheter, the patient reported 10 out of 10 back pain on the numerical rating scale (NRS). The NRS scores at different stages of the surgical procedure are listed in Table 1. After the administration of bupivacaine, the patient reported a significant improvement in his lower back pain (NRS 3-4 out of 10). The placement of the frame and CT scans were completed successfully. The patient denied any significant pain or discomfort and was able to lie flat during the imaging study. He was transported back to the operating room for
TABLE 1. Patient NRS pain score, with 0 being no pain and 10 being worst imaginable pain

\begin{tabular}{lc}
\hline \multicolumn{1}{c}{ Perioperative Stage } & NRS for Pain \\
\hline Preoperative before intrathecal catheter placement & $10 / 10$ \\
\hline During CT scan & $4 / 10$ \\
\hline Intraoperative & $3-6 / 10$ \\
\hline Postoperative & $9 / 10$ \\
\hline
\end{tabular}

DBS stages 1 and 2 procedures. Additional doses of $0.5 \%$ isobaric bupivacaine were administered via an intrathecal catheter. The patient reported low NRS scores (3-4 out of 10) throughout the stage 1 portion of the DBS procedure. The patient was comfortably able to participate throughout the awake procedure. Microelectrode recording and DBS lead placement were successfully performed. The macrocontact of the microelectrode was used for preliminary testing of tremor relief and potential side effects. After DBS lead placement, monopolar review confirmed clinical improvement in the patient's symptoms without any side effects. The patient received no other analgesic or anxiolytic medications.

The stage 2 portion of the DBS procedure was completed immediately after stage 1 under general anesthesia with an endotracheal tube, and the patient was successfully extubated after the completion of the procedure. The patient recovered in the postoperative anesthesia care unit and later was admitted to the neurointensive care unit for postoperative monitoring as per our routine protocol. The patient's postoperative course was uneventful, and he was discharged to home on postoperative day 3 .

\section{Discussion}

\section{Observations}

In this study, we describe the successful use of CSA with a local anesthetic to facilitate the placement of DBS electrodes for Parkinson's disease in a patient with severe low back pain.

First approved in 1996 by the FDA, DBS is indicated for patients with functionally disabling essential tremor or Parkinson's disease with tremors not adequately controlled by medications (https://www.accessdata. fda.gov/scripts/cder/daf/). DBS improves motor function and quality of life for several years in patients with Parkinson's disease. ${ }^{3}$ The success of DBS for symptom management depends on the accurate placement of electrodes, which in turn relies on (1) the patient not taking Parkinson's medications on the day of surgery to facilitate testing, (2) stereotactic magnetic resonance imaging or CT for surgical planning and identifying targets, and (3) intraoperative testing to assess the efficacy of the electrode placement. ${ }^{1}$ Because anesthetic agents can dampen tremors and hinder patient assessment during electrode placement, most DBS surgeries are conducted with the patient awake. ${ }^{1}$ Being restricted to a certain position while awake, however, can be challenging for some patients with Parkinson's disease. Not only do they have a high prevalence $(60 \%)$ of back pain, but they may also have severe dystonia precipitated by withholding their Parkinson's medications. ${ }^{1}$ The top cause of suffering during these cases is immobility. Thus, an important anesthetic consideration is optimizing patient comfort so that the patient can tolerate imaging and intraoperative testing. 


\section{Lessons}

For our patient, chronic severe lumbar back and lower extremity pain was a significant barrier to DBS electrode insertion. Options for back pain management include neuraxial technique or general anesthesia. However, the need for awake testing after DBS lead placement served as a contraindication to general anesthesia. Our decision to use CSA was based on several anesthetic factors and discussions with the neurosurgical team and patient. First, given the patient's history of severe spinal stenosis and previous lumbar surgeries, it was thought that a traditional epidural catheter might be technically difficult to place and might provide inadequate anesthesia and analgesia. ${ }^{5,6}$ With CSA, there is an immediate confirmation of placement with positive CSF drainage. The literature also suggests that CSA has a more rapid onset and denser sensory blockade than epidurals. ${ }^{5}$ Second, the patient had severe cardiac disease, including coronary artery disease, mitral regurgitation, and aortic regurgitation; thus, it was important to use an anesthetic technique that may cause less hemodynamic perturbations. Clinical studies have shown that hemodynamic stability is greater with CSA than with single-shot spinal or epidural anesthesia. ${ }^{5}$ This is due to the possibility of titrating the level of sensory/motor block with small incremental doses of local anesthetic until adequate block is achieved. Finally, neuraxial anesthesia facilitated both pain management and the surgical requirement of avoiding any alteration or suppression of the patient's arousal and baseline tremor in the upper extremities during macrosimulation testing.

Lotto et al. ${ }^{7}$ also used neuraxial techniques to manage two patients with Parkinson's disease with back pain who were undergoing DBS surgeries, but they instead used intrathecal opioids to manage the back pain rather than local anesthetics as in our situation. Their only documented issue was the lack of efficacy when a bolus dose of intrathecal fentanyl was administered through a continuous spinal catheter. The issue was resolved with the use of intrathecal hydromorphone. Although their approach has the advantage of avoiding lower extremity paralysis, we elected to use a local anesthetic (bupivacaine) over opioids for several reasons. We thought that it was easier to titrate CSA using local anesthetics with better predictability of onset and offset and simpler assessment methods to determine efficacy (to pain and temperature) than opioids. In addition, the use of intrathecal hydromorphone has not been approved by the FDA. Furthermore, the intrathecal administration of more hydrophilic opioids such as hydromorphone may have rostral spread and lead to sedation, delayed respiratory depression, and perioperative delirium. ${ }^{7,8}$ This may confuse the diagnosis of other severe postoperative complications of DBS surgery, such as intracranial hemorrhage, and may require more intense postoperative monitoring. Consequently, we thought that CSA with local anesthetic was the more appropriate option for our patient, who in particular was undergoing DBS surgery for upper extremity parkinsonian symptoms.

Another option would be general anesthesia. Asleep DBS surgeries using stereotactic imaging and microelectrode recordings have been performed in patients not suitable for awake procedures (i.e., unbearable motor or nonmotor off-medication symptoms and anxiety). ${ }^{9}$ However, this option was not feasible because of the surgeon's desire for an alert patient who could be assessed intraoperatively.
In conclusion, we report an effective strategy to manage patients with severe back pain undergoing DBS surgery for upper extremity motor symptoms. The CSA technique with a local anesthetic may allow a greater range of patients to tolerate awake DBS electrode placement in the setting of severe back and lower extremity pain.

\section{References}

1. Poon CC, Irwin MG. Anaesthesia for deep brain stimulation and in patients with implanted neurostimulator devices. $\mathrm{Br} J$ Anaesth. 2009;103(2):152-165.

2. Smith $H$, Gee L, Kumar V, et al. Deep brain stimulation significantly decreases disability from low back pain in patients with advanced Parkinson's disease. Stereotact Funct Neurosurg. 2015;93(3): 206-211.

3. Limousin P, Foltynie T. Long-term outcomes of deep brain stimulation in Parkinson disease. Nat Rev Neurol. 2019;15(4):234-242.

4. Chevrier E, Fraix V, Krack $P$, et al. Is there a role for physiotherapy during deep brain stimulation surgery in patients with Parkinson's disease? Eur J Neurol. 2006;13(5):496-498.

5. Veličković I, Pujic B, Baysinger CW, Baysinger CL. Continuous spinal anesthesia for obstetric anesthesia and analgesia. Front Med (Lausanne). 2017;4:133.

6. Sharrock NE, Urquhart B, Mineo R. Extradural anaesthesia in patients with previous lumbar spine surgery. $\mathrm{Br} J$ Anaesth. 1990;65(2):237-239.

7. Lotto M, Boulis NM. Intrathecal opioids for control of chronic low back pain during deep brain stimulation procedures. Anesth Analg. 2007;105(5):1410-1412.

8. American Society of Anesthesiologists. Practice guidelines for the prevention, detection, and management of respiratory depression associated with neuraxial opioid administration: an updated report by the American Society of Anesthesiologists Task Force on Neuraxial Opioids and the American Society of Regional Anesthesia and Pain Medicine. Anesthesiology. 2016;124(3):535-552.

9. Blasberg F, Wojtecki L, Elben S, et al. Comparison of awake vs. asleep surgery for subthalamic deep brain stimulation in Parkinson's disease. Neuromodulation. 2018;21(6):541-547.

\section{Disclosures}

The authors report no conflict of interest concerning the materials or methods used in this study or the findings specified in this paper.

\section{Author Contributions}

Conception and design: Kunkler, Patil, Chiravuri. Acquisition of data: Tarnal, Kunkler, Patil. Analysis and interpretation of data: Tarnal, Kunkler, Patil. Drafting the article: all authors. Critically revising the article: all authors. Reviewed submitted version of manuscript: all authors. Approved the final version of the manuscript on behalf of all authors: Tarnal. Study supervision: Patil.

\section{Correspondence}

Vijay Tarnal: University of Michigan Medical School, Ann Arbor, Ml. vtarnal@med.umich.edu. 